\title{
Anomalous metallic state above the upper critical field of the conventional three-dimensional superconductor $\mathrm{AgSnSe}_{2}$ with strong intrinsic disorder
}

\author{
Zhi Ren, M. Kriener, A. A. Taskin, Satoshi Sasaki, Kouji Segawa, and Yoichi Andd* \\ Institute of Scientific and Industrial Research, Osaka University, Ibaraki, Osaka 567-0047, Japan
}

(Dated: August 31, 2018)

\begin{abstract}
We report superconducting properties of $\mathrm{AgSnSe}_{2}$ which is a conventional type-II superconductor in the very dirty limit due to intrinsically strong electron scatterings. While this material is an isotropic three-dimensional (3D) superconductor with a not-so-short coherence length where strong vortex fluctuations are not expected, we found that the magnetic-field-induced resistive transition at fixed temperatures becomes increasingly broader toward zero temperature and, surprisingly, that this broadened transition is taking place largely above the upper critical field determined thermodynamically from the specific heat. This result points to the existence of an anomalous metallic state possibly caused by quantum phase fluctuations in a strongly-disordered 3D superconductor.

PACS numbers: 74.62.En, 74.40.-n, 74.25.Dw, 74.70.Dd
\end{abstract}

\section{INTRODUCTION}

The interplay between disorder and superconductivity has attracted sustained interest over the past few decades. In BCS superconductors, according to Anderson's theorem, $\frac{1}{\underline{1}}$ the superconducting critical temperature $T_{\mathrm{c}}$ and the energy gap $\Delta$ remain unaffected by the presence of weak disorder, although in unconventional superconductors both $T_{\mathrm{c}}$ and $\Delta$ are strongly suppressed with weak disorder $\stackrel{2}{2}$ In the strongly disordered regime, things become interesting even in BCS superconductors: In two-dimensional (2D) systems, strong disorder leads to a universal superconductor-to-insulator transition at a critical sheet resistance of $\sim h / 4 e^{2}, \underline{3-10}$ furthermore, it has been found that, as $T$ tends to zero, there emerges a broad range of magnetic field where the resistivity remains finite but is much smaller than the normal-state value, which arguably signifies the importance of quantum phase fluctuations, $\frac{11,12}{1 n}$ three-dimensional (3D) systems, strong disorder leads to a disruption of the global superconducting coherence through reductions in both the superfluid density $n_{\mathrm{s}}$ and pairing interactions, which results in the superconducting order parameter to spatially fluctuate and gives rise to anomalous electronic states near the superconductor-to-metal transition. $\underline{13,14}$ Experimentally, while the 2D systems have been actively studied in the past, $\underline{\underline{3}-12,15} \underline{\underline{21}}$ the role of strong disorder in $3 \mathrm{D}$ superconductors is just beginning to be addressed with a modern viewpoint, 22,23 which naturally calls for explorations of suitable materials for such a study.

In this paper, we report that $\mathrm{AgSnSe}_{2}$, which is a low- $T_{c}$ conventional superconductor having a cubic structure, offers an ideal playground for studying the effects of strong disorder in 3D superconductors. We show that one can synthesize high-quality samples of $\mathrm{AgSnSe}_{2}$ presenting a very sharp superconducting transition, which indicates that morphologically the system is homogeneous; nonetheless, strong electron scatterings that are intrinsic to this system lead to an extremely type-II superconductivity with the Ginzburg-Landau parameter $\kappa_{\mathrm{GL}}$ of as large as 55. This causes the emergence of a broad range of magnetic fields where the resistivity remains finite but is much smaller than the normal-state value as $T \rightarrow 0$, which is very much reminiscent of the behavior observed in disordered $2 \mathrm{D}$ superconductors 11,12 or in quasi-2D systems like high- $T_{c}$ cuprates $\stackrel{24,25}{ }$ Furthermore, such an anomalous metallic state is found in the magnetic field range above the upper critical field determined thermodynamically from the specific heat. Since this anomalous state can be easily reached with the magnetic field of 3 $\mathrm{T}$ and the microscopic mechanism of superconductivity is well understood in conventional superconductors, future studies of $\mathrm{AgSnSe}_{2}$ would help elucidate the roles of quantum phase fluctuations which remain controversial to date $\stackrel{11-23}{-23}$

\section{II. $\mathrm{AgSnSe}_{2} \mathrm{SUPERCONDUCTOR}$}

The superconductivity in $\mathrm{AgSnSe}_{2}$ was discovered by Johnston and Adrian $^{26}$ in 1977 as a possible valenceskipping superconductor. In valence-skipping compounds, the nominal valence state of a constituent element happens to be the skipped valence for that element. This characteristic has been of particular interest for superconductivity, since it may provide a means to realize the so-called negative- $U$ mechanism to enhance the superconducting transition temperature $T_{c} \stackrel{27}{2}$ Well-known examples of such valence-skipping superconductors are $\mathrm{BaBi}_{1-x} \mathrm{~Pb}_{x} \mathrm{O}_{3} \stackrel{28}{\stackrel{28}{\rightleftharpoons}} \mathrm{Ba}_{1-x} \mathrm{~K}_{x} \mathrm{BiO}_{3} \stackrel{29}{\stackrel{2}{=}}$ and $\mathrm{Pb}_{1-x} \mathrm{Tl}_{x} \mathrm{Te}_{2} \stackrel{30}{\underline{2}}$ In all these compounds, $T_{c}$ is relatively high for their low carrier density. At the same time, valence fluctuations lead to significant electron scattering, making those superconductors highly disordered even though the matrix is homogeneous.

$\mathrm{AgSnSe}_{2}$ adopts the face-centered cubic structure, in which the cation sites are randomly occupied by equal amount of $\mathrm{Ag}$ and $\mathrm{Sn}$ atoms. Since the nominal valence of $\mathrm{Sn}$ is $3+$ which coincides with its skipped valence state, the $\mathrm{Sn}$ ions should be separated into 1:1 mixture of $\mathrm{Sn}^{2+}$ and $\mathrm{Sn}^{4+}$ and the chemical formula of this material can be more appropriately expressed 

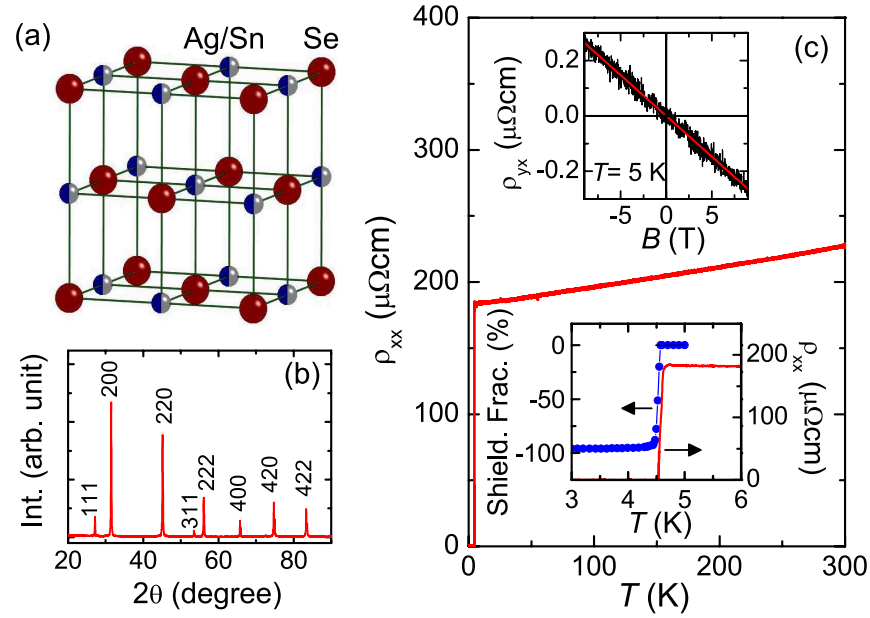

FIG. 1: (Color online) (a) Face-centered cubic structure of $\mathrm{AgSnSe}_{2}$. Note that $\mathrm{Ag}$ and $\mathrm{Sn}$ atoms are randomly distributed in the cation sites. (b) XRD pattern of the $\mathrm{AgSnSe}_{2}$ sample. All the diffraction peaks can be well indexed using a cubic cell with the Fm3m space group. (c) Temperature dependence of $\rho_{x x}$ of the $\mathrm{AgSnSe}_{2}$ sample. Lower inset shows an enlarged view of the data near the superconducting transition for $\rho_{x x}$ (right axis) and for the dc magnetic susceptibility measured upon zero-field-cooling (left axis, shown in terms of the shielding fraction). Upper inset shows the magnetic-field dependence of $\rho_{y x}$ at $5 \mathrm{~K}$; its slope gives $n_{e}=2.0 \times 10^{22}$ $\mathrm{cm}^{-3}$.

as $\left(\mathrm{Ag}^{1+}\right)\left(\mathrm{Sn}^{2+}\right)_{0.5}\left(\mathrm{Sn}^{4+}\right)_{0.5}\left(\mathrm{Se}^{2-}\right)_{2}$, as evidenced by the measurements of the magnetic susceptibility ${ }^{26}$ and the ${ }^{119} \mathrm{Sn}$ Mössbauer spectra $\stackrel{31}{{ }^{31}}$ Despite the valence skipping nature and the carrier density of the order of $10^{22} \mathrm{~cm}^{-3}$, its $T_{c}$ of $4.7 \mathrm{~K}$ is not particularly high, which suggests that the negative- $U$ mechanism is not at work here. Therefore, in this compound one can explore manifestations of strong disorder associated with valence fluctuations in the context of conventional superconductivity without being bothered by strong electron interactions.

\section{EXPERIMENTAL}

We synthesized high-quality ingots of polycrystalline $\mathrm{AgSnSe}_{2}$ by using a two-step method as described in Ref. 26. First, high-purity shots of Ag (99.999\%), Sn (99.99\%), and Se (99.999\%) with the stoichiometric ratio of 1:1:2 were melted in sealed evacuated quartz tube at $800{ }^{\circ} \mathrm{C}$ for $48 \mathrm{~h}$ with intermittent shaking to ensure homogeneity, followed by quenching to room temperature. The quartz tube containing the melt-quenched ingot was subsequently annealed at $450{ }^{\circ} \mathrm{C}$ for two weeks, and then quenched into cold water. The structure of the resulting sample was characterized by powder X-ray diffraction (XRD). As can be seen in Fig. 1(b), the XRD pattern shows sharp diffraction peaks consistent with the facecentered cubic structure, giving the lattice parameter $a$ of 5.675(1) $\AA$ which agrees with the literature $\underline{\underline{26}}$
The ingot was cut into bar-shaped samples, and the temperature-dependent magnetization $M$ under various magnetic fields was measured with a vibrating sample magnetometer (VSM), while the magnetization curves at fixed temperatures were measured with a commercial SQUID magnetometer (Quantum Design MPMS- 1). The demagnetization effect was corrected for by considering the slab geometry ${ }^{32}$ The resistivity $\rho_{x x}$ and the Hall resistivity $\rho_{y x}$ were measured by using a standard six-probe method where the contacts were made by spotwelding gold wires. The specific heat $c_{p}$ was measured with a relaxation-time method using Quantum Design PPMS-9. For consistency reasons, all the data presented here were measured on exactly the same sample.

\section{BASIC CHARACTERIZATIONS}

\section{A. Transport properties and superconducting transition}

Figure 1(c) shows the temperature dependence of $\rho_{x x}$, which exhibits only a weak metallic temperature dependence. This is qualitatively similar to that reported ${ }^{26}$ for $\mathrm{Ag}_{0.76} \mathrm{Sn}_{1.24} \mathrm{Se}_{2}$ and signifies the presence of intrinsically strong disorder that does not change much with the $\mathrm{Ag} / \mathrm{Sn}$ ratio. As shown in the upper inset of Fig. 1(c), $\rho_{y x}$ is linear in $B$ and its slope at $5 \mathrm{~K}$ corresponds to an electron density $n_{\mathrm{e}}$ of $2.0 \times 10^{22} \mathrm{~cm}^{-3}$. The superconducting transition in $\rho_{x x}$ occurs sharply between 4.5 and $4.6 \mathrm{~K}$, and the magnetic susceptibility shows the onset $T_{c}$ of $4.55 \mathrm{~K}$, which corresponds to the midpoint of the resistive transition. The superconducting shielding faction achieves nearly $100 \%$ after the demagnetization correction. There is no resistivity upturn at low temperatures, giving no clear sign of Anderson localization.

\section{B. Specific heat}

Figure 2(a) shows the temperature dependences of $c_{p} / T$ measured under 0 and $9 \mathrm{~T}$. Application of a 9 T field completely suppresses the superconductivity, enabling us to determine the phononic contribution and the normal-state parameters such as the electronic specificheat coefficient $\gamma_{\mathrm{n}}$ and the effective mass $m^{*} \underline{\underline{33}}$ Figure 2(b) shows the temperature dependence of the electronic specific heat $c_{\mathrm{el}} / T$ in $0 \mathrm{~T}$ obtained by subtracting the phononic contribution determined from the 9-T data. One can see that $c_{\mathrm{el}} / T$ quickly approaches zero at low $T$, indicating a fully-gapped nature. As for the precise temperature dependence, although the simple weak-coupling BCS model (dash-dotted line) fails to describe the $c_{\mathrm{el}} / T$ data, a modified BCS model ${ }^{34}$ that allows the coupling constant $\alpha \equiv \Delta(0) / k_{\mathrm{B}} T_{\mathrm{c}}$ to vary $[\Delta(0)$ is the gap at $0 \mathrm{~K}]$ can fit our data well with $\alpha=1.925$ (solid line), which is only slightly larger than the weak-coupling BCS value of 1.764 . 

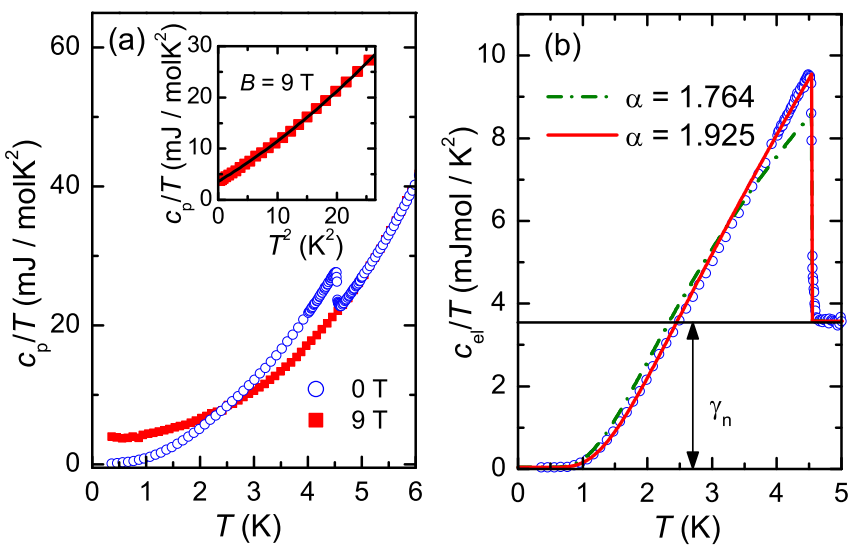

FIG. 2: (Color online) (a) Temperature dependences of $c_{p} / T$ measured in 0 and $9 \mathrm{~T}$. Inset shows $c_{p} / T$ plotted as a function of $T^{2}$ for the 9-T data, where the superconductivity is completely suppressed; the solid line presents the best fit to the data using the Debye model (see Ref. 33). (b) Temperature dependence of $c_{\mathrm{el}} / T$ in $0 \mathrm{~T}$. The dash-dotted line is the calculated $c_{\mathrm{el}} / T$ curve given by the weak-coupling BCS theory; the solid line is the best fit using a modified BCS theory with $\alpha=1.925$. The horizontal solid line denotes $\gamma_{\mathrm{n}}$.

Using $m^{*}$ and $\alpha$ obtained from $c_{\mathrm{p}}(T) \stackrel{33}{\underline{3}}$ the Pippard coherence length $\xi_{0}$ can be estimated as $\xi_{0}=$ $\hbar^{2}\left(3 \pi^{2} n_{e}\right)^{1 / 3} /\left(\pi \alpha k_{\mathrm{B}} T_{\mathrm{c}} m^{*}\right)=181 \mathrm{~nm}$. This is to be contrasted with the mean free path $\ell=\hbar\left(3 \pi^{2}\right)^{1 / 3} /\left(\rho_{0} n_{e}^{2 / 3} e^{2}\right)$ $=0.9 \mathrm{~nm}$, where $\rho_{0}$ is the residual resistivity. This $\ell$ is less than $2 a$ and is very short, pointing to the existence of strong scattering which is consistent with the valence fluctuations of $\mathrm{Sn}$. The ratio $l / \xi_{0}=0.005$ means that $\mathrm{AgSnSe}_{2}$ is in the very dirty limit. Yet, $k_{\mathrm{F}} \ell$ is estimated to be $\sim 8$ and hence the system is still a good metal. Note that the estimations of $m^{*}, k_{\mathrm{F}}, \xi_{0}$, and $\ell$ in this paper are based on the free electron theory; once the band structure of $\mathrm{AgSnSe}_{2}$ is known, those estimations can be made more accurate.

\section{Critical fields}

Figure 3 summarizes the results of the magnetization measurements to extract the values of the upper and lower critical fields. Figure 3(a) shows $M(B)$ curves measured after zero-field cooling to various temperatures. We define $B_{1}$ at each temperature as the value at which the $M(B)$ data deviates from its initial linear behavior, as can be more clearly seen in the inset of Fig. 3(b). Extrapolation ${ }^{35}$ of the resulting $B_{1}(T)$ data to $T=0 \mathrm{~K}$ yields $B_{1}(0)=1.18 \mathrm{mT}$ which, after correcting for the demagnetization effect,, 32 gives the lower critical field at $T=0 \mathrm{~K}, B_{\mathrm{c} 1}(0)$, of $1.58 \mathrm{mT}$.

Figure $3(\mathrm{c})$ shows the temperature dependences of the magnetization near the superconducting transition under various magnetic fields. A paramagnetic background with a temperature-independent susceptibility $\chi_{0}=4.2$
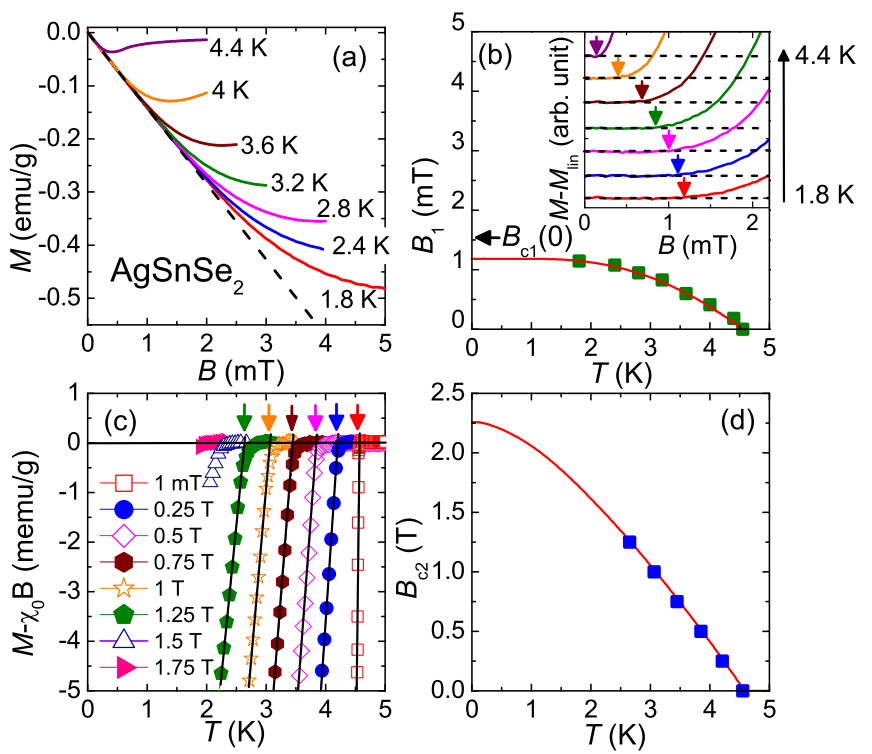

FIG. 3: (Color online) (a) $M(B)$ curves measured after zerofield cooling to various temperatures. The dashed line marks the initial linear behavior. (b) Plot of $B_{1}$ vs $T$; the solid line is a fit to the data using the local dirty limit formula. The $B_{\mathrm{c} 1}(0)$ obtained after the demagnetization correction is marked by an arrow. Inset shows the $B$ dependences of $\Delta M \equiv M-M_{\text {lin }}$, where $M_{\text {lin }}$ denotes the initial Meissner contribution; the data are shifted vertically for clarity. The determined $B_{1}$ value for each temperature is shown by arrows. (c) Temperature dependences of $M_{\mathrm{sc}} \equiv M-\chi_{0} B$ under various magnetic fields. The $T_{\mathrm{c}}^{\mathrm{Mag}}(B)$ values are indicated by arrows. (d) $B_{\mathrm{c} 2}^{\mathrm{Mag}}$ vs $T$ phase diagram; the solid line is a WHH fit to the data.

$\times 10^{-8} \mathrm{emu} / \mathrm{g}$ has been subtracted from the data,$\underline{36}$ and what is plotted is $M_{\mathrm{sc}} \equiv M-\chi_{0} B$. A gradual suppression of superconductivity with increasing magnetic field is evident. For each magnetic field, we determined the onset temperature of the transition, $T_{\mathrm{c}}^{\mathrm{Mag}}(B)$, from the intersection of the linear extrapolation of the $M_{\mathrm{sc}}(T)$ data to the $M_{\mathrm{sc}}=0$ line, as indicated by the arrows in Fig. 3(c). The obtained $T_{\mathrm{c}}^{\mathrm{Mag}}(B)$ data shown in Fig. 3(d) give the temperature dependence of the magnetically-determined upper critical field, $B_{c 2}^{\mathrm{Mag}}(T)$, which is extrapolated to $T$ $=0 \mathrm{~K}$ using the Werthamer-Helfand-Hohenberg $(\mathrm{WHH})$ theory $\stackrel{37}{3}$ to yield $B_{\mathrm{c} 2}^{\mathrm{Mag}}(0)=2.26 \mathrm{~T}$.

To evaluate the upper critical field at low temperatures in a more direct way, we measured the superconducting transition in specific heat under various magnetic fields. Figure 4(a) shows representative data of $c_{\mathrm{el}} / T$ vs $T$ measured in $0,0.25,1.0$, and $1.5 \mathrm{~T}$, where the superconducting transition is well resolved. In higher magnetic fields, the specific-heat anomaly becomes weaker, as shown in Fig. 4(b) for 1.75, 1.9, and 2.0 T; here, the transition can still be seen in $1.9 \mathrm{~T}$, but no clear anomaly is discernible at $2.0 \mathrm{~T}$. These data demonstrate that the upper critical field is thermodynamically well-defined in $\mathrm{AgSnSe}_{2}$ at low temperature and that it lies around $2 \mathrm{~T}$ near $T=$ $0 \mathrm{~K}$. 

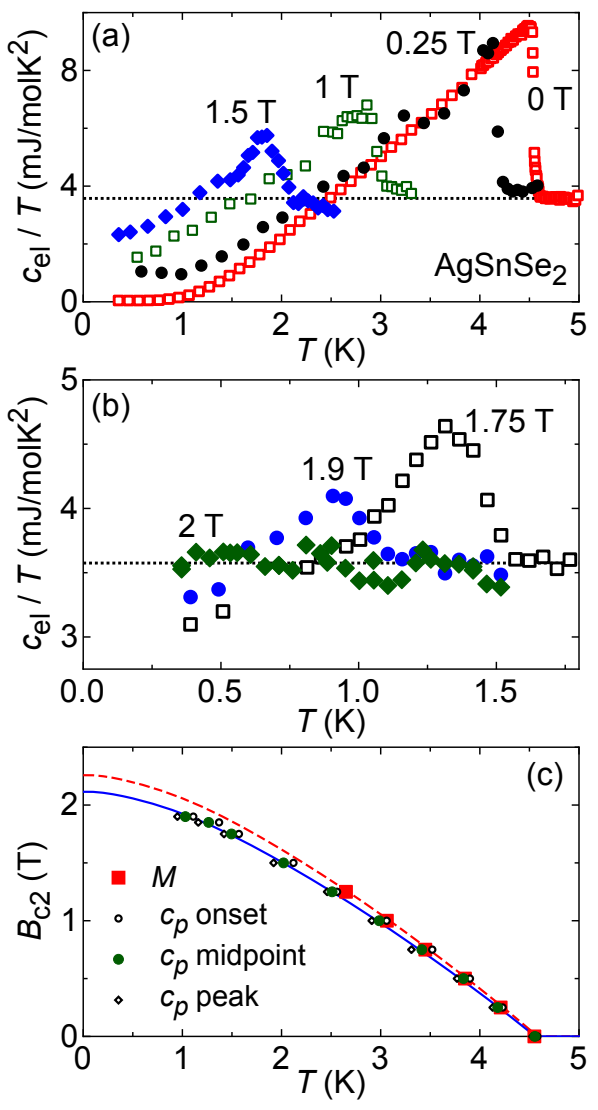

FIG. 4: (Color online) (a, b) Superconducting transition measured in the temperature dependence of the specific heat under various magnetic fields. (c) $B_{c 2}^{C p}$ vs $T$ phase diagram; the solid line is a WHH fit to the data. For comparison, $B_{c 2}^{\mathrm{Mag}}(T)$ and its WHH fitting are shown with filled squares and a dashed line, respectively.

To quantitatively analyze the superconducting transition in the specific-heat data, for each magnetic field we determined three characteristic temperatures: onset of the transition (below which the specific heat grows), midpoint of the transition, and the peak in $c_{p}(T)$. It is useful to note that the transition width $\Delta T_{c}^{C p}$, which we define by the difference between the onset and peak temperatures, remains reasonably narrow with $\Delta T_{c}^{C p} \lesssim 0.3$ $\mathrm{K}$ at all temperatures. We choose to use the midpoint of the specific-heat transition for defining the transition temperature $T_{\mathrm{c}}^{C p}(B)$ for each magnetic field, which in turn gives the temperature dependence of the upper critical field $B_{c 2}^{C p}(T)$.

The solid line in Fig. 4(c) is the fitting of the WHH theory to the $B_{c 2}^{C p}(T)$ data, which gives the $T=0 \mathrm{~K}$ value $B_{c 2}^{C p}(0)$ of $2.11 \mathrm{~T}$. For comparison, we reproduced in Fig. $4(\mathrm{c})$ the $B_{c 2}^{\mathrm{Mag}}(T)$ data obtained from the magnetization measurements. The difference in the WHH fittings for the specific-heat and magnetization data is only $7 \%$, which indicates that the two thermodynamical measurements are essentially consistent. In the following discussions, we take $B_{c 2}^{C p}$ as the thermodynamically-

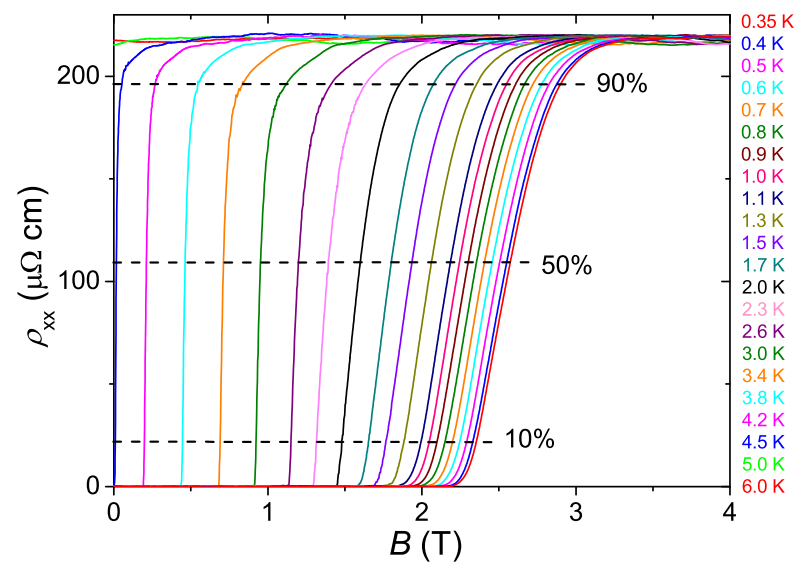

FIG. 5: (Color online) Magnetic-field-induced resistive transition measured at various temperatures. The level of $\rho_{x x}$ that is used for determining $B_{10 \%}, B_{50 \%}$, and $B_{90 \%}$ are indicated by dashed lines.

determined $B_{c 2}$.

\section{Superconducting parameters}

Now we estimate various superconducting parameters of $\mathrm{AgSnSe}_{2}$ by using $B_{\mathrm{c} 2}^{C p}(0)$ as the zero-temperature upper critical field $B_{\mathrm{c} 2}(0)$. Note that since $\mathrm{AgSnSe}_{2}$ has cubic symmetry, no anisotropy is expected. The Ginzburg-Landau (GL) coherence length $\xi_{\mathrm{GL}}(0)$ is estimated as $\xi_{\mathrm{GL}}(0)=\sqrt{\Phi_{0} / 2 \pi B_{\mathrm{c} 2}(0)}=12.5 \mathrm{~nm}$, where $\Phi_{0}=2.07 \times 10^{-15} \mathrm{~Wb}$ is the flux quantum. Alternatively, one may also estimate $\xi_{\mathrm{GL}}$ from $\xi_{\mathrm{GL}}(0) \simeq \sqrt{\xi_{0} \ell}$ for dirty superconductors, which yields $\xi_{\mathrm{GL}} \simeq 12.7 \mathrm{~nm}$. The consistency is gratifying. The equation $38 B_{\mathrm{c} 1}(0) / B_{\mathrm{c} 2}(0)$ $=\left(\ln \kappa_{\mathrm{GL}}+0.5\right) /\left(2 \kappa_{\mathrm{GL}}^{2}\right)$ gives the $\mathrm{GL}$ parameter $\kappa_{\mathrm{GL}}$ $=55$, which in turn allows us to calculate the effective penetration depth $\lambda_{\text {eff }}=685 \mathrm{~nm}$ through $B_{\mathrm{c} 1}(0)=$ $\left(\Phi_{0} / 4 \pi \lambda_{\text {eff }}^{2}\right)\left(\ln \kappa_{\mathrm{GL}}+0.5\right)$. This gives the London penetration depth $\lambda_{\mathrm{L}} \simeq \lambda_{\text {eff }} \sqrt{\ell / \xi_{0}}=48.3 \mathrm{~nm}$.

Since $\mathrm{AgSnSe}_{2}$ is a very dirty superconductor, the superfluid density $n_{s}$ is expected to be reduced by a factor of $\sim \ell / \xi_{0}{ }^{39}$ Indeed, the calculation of $n_{s}$ based on $n_{s}=$ $m^{*} /\left(\mu_{0} e^{2} \lambda_{\text {eff }}^{2}\right)$ yields $n_{s} \approx 9 \times 10^{19} \mathrm{~cm}^{-3}$, which is close to the estimation of $n_{s} \approx n_{\mathrm{e}}\left(\ell / \xi_{0}\right)=1 \times 10^{20} \mathrm{~cm}^{-3}$, reassuring the applicability of the BCS theory to $\mathrm{AgSnSe}_{2}$ and the soundness of our $B_{c 1}$ measurements which can be adversely affected by flux pinning.

\section{ANOMALOUS METALLIC STATE}

\section{A. Resistive transition in magnetic fields}

Despite the conventional nature of the superconductivity in $\mathrm{AgSnSe}_{2}$, nontrivial physics becomes evident when 


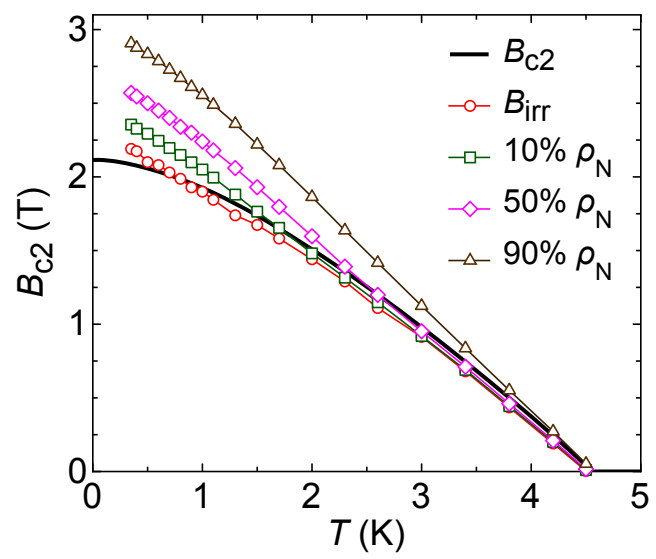

FIG. 6: (Color online) Plots of $B_{c 2}$ determined thermodynamically from specific-heat measurements (solid line) together with $B_{\text {irr }}, B_{10 \%}, B_{50 \%}$, and $B_{90 \%}$ obtained from the resistive transition data.

one looks at the magnetic-field-induced resistive transition at fixed temperatures (Fig. 5). One can easily see that the resistive transition is very sharp at temperatures close to $T_{c}$, but it becomes increasingly broader at lower temperatures. We determined the values of the magnetic field at which $\rho_{x x}$ recovers a certain percentage of the normal-state resistivity $\rho_{\mathrm{N}}$ for each temperature, and denote them $B_{10 \%}(T), B_{50 \%}(T)$, etc. We also determined the irreversibility field $B_{\text {irr }}(T)$ to mark the onset of a finite resistivity within our sensitivity limit, which corresponds to $0.15 \%$ of $\rho_{\mathrm{N}}$. The results are shown in Fig. 6, where we also plotted the thermodynamicallydetermined $B_{c 2}(T)$ with a solid line.

Usually, either $B_{50 \%}(T)$ or $B_{90 \%}(T)$ are considered to give a measure of the upper critical field $\stackrel{40,41}{t n}$ the present case, for temperatures down to $T / T_{c} \sim 1 / 2$, $B_{50 \%}(T)$ agrees reasonably well with the thermodynamically determined $B_{c 2}$, but at lower temperatures the resistive transition occurs largely above $B_{c 2}$. This means that at low temperature, there is a wide range of magnetic field above $B_{c 2}$ where the resistivity is finite but is noticeably smaller than $\rho_{\mathrm{N}}$. The existence of such an anomalous metallic state above $B_{c 2}$ has never been documented for a conventional 3D superconductor, and this is the main finding of the present work. Note that the Pauli paramagnetic limit is estimated to be $8.4 \mathrm{~T}$, which is well above the anomalous metallic regime observed here.

Similar broadening of the magnetic-field-induced resistive transition at low temperatures has been observed in high-temperature superconductors such

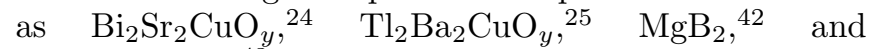
$\mathrm{Ba}_{1-x} \mathrm{~K}_{x} \mathrm{BiO}_{3} \stackrel{43}{\stackrel{4}{*}}$ This phenomenon is generally believed to be caused by enhanced vortex fluctuations and the resulting vortex liquid phase due to a short coherence length that is inherent to high-temperature superconductors. In light of this common belief, the present observation in $\mathrm{AgSnSe}_{2}$ is striking. Here the coherence length is not so short, $12.1 \mathrm{~nm}$, and the system is $3 \mathrm{D}$, both of which work against strong vortex fluctuations. Therefore, our result calls for a reinterpretation of the low-temperature broadening of the magnetic-field-induced resistive transition.

\section{B. Quantum phase fluctuations}

To understand the above results, an interesting possibility is the scenario proposed by Spivak, Oreto and Kivelson 13 that the strong disorder leads to formations of superconducting "puddles" that are Josephson-coupled to each other, and quantum fluctuations of the relative phase between those puddles give rise to a broad range of magnetic fields where the resistivity remains finite and smaller than $\rho_{\mathrm{N}}$ as $T \rightarrow 0$. If this is indeed the case, our data give evidence for the important role of quantum phase fluctuations in disordered 3D conventional superconductors. Since the superconductivity mechanism is well understood here (as opposed to the case of high-temperature superconductors) and the required magnetic field to access the quantum fluctuation regime is low (only $\sim 3 \mathrm{~T}$ ), $\mathrm{AgSnSe}_{2}$ provides a convenient platform for studying the role of quantum fluctuations in the magnetic-field-induced superconductor-tometal transition.

Another anomalous behavior observed here is that at low temperatures, not only $B_{90 \%}$ but also $B_{\text {irr }}$ show little tendency toward saturation. A similar behavior was previously observed in strongly-disordered amorphous superconductors $\stackrel{41}{ }$ and was discussed to be due to the weak-localization effect $\underline{\underline{44}}$ However, the discrepancy between the resistive transition and the thermodynamically-determined $B_{c 2}$ makes the conclusion of those old studies ${ }^{41,44}$ questionable. Hence, the physical meaning of the resistive transition in disordered superconductors had better be reconsidered by taking into account the important role of quantum phase fluctuations.

\section{SUMMARY}

We have fully characterized the $\mathrm{AgSnSe}_{2}$ superconductor where its valence-skipping nature leads to intrinsically strong electron scattering without any obvious enhancement of $T_{c}$. At low temperature, we observed an anomalous broadening of the magnetic-fieldinduced resistive transition which occurs largely above the thermodynamically-determined $B_{c 2}$. This means the existence of an anomalous metallic state above $B_{c 2}$ where quantum phase fluctuations associated with a magnetic-field-induced superconductor-to-metal transition are likely to be playing an important role. This makes $\mathrm{AgSnSe}_{2}$ a useful platform for studying the role of quantum fluctuations in 3D disordered superconductors. 


\section{Acknowledgments}

We thank S. A. Kivelson for illuminating discussions. This work was supported by JSPS (NEXT Program),
MEXT (Innovative Area "Topological Quantum Phenomena" KAKENHI 22103004), and AFOSR (AOARD 124038).
* Electronic address: yªndo@sanken.osaka-u.ac.jp

1 P. W. Anderson, J. Phys. Chem. Solids 11, 26 (1959).

2 R. Balian and N. R. Werthamer, Phys. Rev. 131, 1553 (1963).

3 M. P. A. Fisher, Phys. Rev. Lett. 65, 923 (1990).

4 D. B. Haviland, Y. Liu, and A. M. Goldman, Phys. Rev. Lett. 62, 2180 (1989).

5 A. F. Hebard and M. A. Paalanen, Phys. Rev. Lett. 65, 927 (1990).

${ }^{6}$ M. A. Paalanen, A. F. Hebard, and R. R. Ruel, Phys. Rev. Lett. 69, 1604 (1992).

7 S. Tanda, S. Ohzeki, and T. Nakayama, Phys. Rev. Lett. 69, 530 (1992).

8 M. P. A. Fisher and D. H. Lee, Phys. Rev. B 39, 2756 (1989).

9 T. Pang, Phys. Rev. Lett. 62, 2176 (1989).

10 M. P. A. Fisher, G. Grinstein, and S. M. Girvin, Phys. Rev. Lett. 64, 587 (1990).

11 N. Mason and A. Kapitulnik, Phys. Rev. Lett. 82, 5341 (1999).

12 M. A. Steiner, G. Boebinger, and A. Kapitulnik, Phys. Rev. Lett. 94, 107008 (2005).

13 B. Spivak, P. Oreto, and S. A. Kivelson, Phys. Rev. B 77, 214523 (2008).

14 M. V. Feigel'man, L. B. Ioffe, V. E. Kravtsov, E. Cuevas, Ann. Phys. 325, 1368 (2010).

15 J. M. Valles, Jr., R. C. Dynes, and J. P. Garno, Phys. Rev. Lett. 69, 3567 (1992).

16 Y. Liu, D. B. Haviland, B. Nease, and A. M. Goldman, Phys. Rev. B 47, 5931 (1993).

17 S. Doniach, J. Phys. Chem. Solids, 56, 1601 (1995).

18 G. Sambandamurthy, L. W. Engel, A. Johansson, and D. Shahar, Phys. Rev. Lett. 92, 107005 (2004).

19 Y. Dubi, Y. Meir, and Y. Avishai, Nature 449, 876 (2007).

${ }^{20}$ M. D. Stewart Jr., A. J. Yin, J. M. Xu, and J. M. Valles Jr., Science 318, 1273 (2007).

21 B. Sacepe, T. Dubouchet, C. Chapelier, M. Sanquer, M. Ovadia, D. Shahar, M. Feigel'man, and L. Ioffe, Nature Phys. 7, 239 (2011).

22 M. Mondal, S. Kumar, M. Chand, A. Kamlapure, G. Saraswat, G. Seibold, L. Benfatto, and P. Raychaudhuri, Phys. Rev. Lett. 107, 217003 (2011).

23 M. Chand, G. Saraswat, A. Kamlapure, M. Mondal, S. Kumar, J. Jesudasan, V. Bagwe, L. Benfatto, V. Tripathi, and P. Raychaudhuri, Phys. Rev. B 85, 014508 (2012).

24 M. S. Osofsky, R. J. Soulen, Jr., S. A. Wolf, J. M. Broto, H. Rakoto, J. C. Ousset, G. Coffe, S. Askenazy, P. Pari, I. Bozovic, J. N. Eckstein, and G. F. Virshup, Phys. Rev. Lett. 71, 2315 (1993).

25 A. P. Mackenzie, S. R. Julian, G. G. Lonzarich, A. Carrington, S. D. Hughes, R. S. Liu, and D. C. Sinclair, Phys. Rev. Lett. 71, 1238 (1993)
${ }^{26}$ D. C. Johnston and H. Adrian, J. Phys. Chem. Solids 38, 355 (1977).

27 C. M. Varma, Phys. Rev. Lett. 61, 2713 (1988).

28 A. W. Sleight, J. L. Gillson, and P. E. Bierstedt, Solid State Commu. 17, 27 (1975).

29 R. J. Cava, B. Batlogg, J. J. Krajewski, R. Farrow, L. W. Rupp Jr, A. E. White, K. Short, W. F. Peck, and T. Kometani, Nature 332, 814 (1988).

30 S. A. Nemov and Y. I. Ravich, Phys. Uep. 41, 735 (1998).

31 F. S. Nasredinov, S. A. Nemov, V. F. Masterov, and P. P. Seregin, Phys. Solid State 41, 1741 (1999).

32 E. H. Brandt, Phys. Rev. B 60, 11939 (1999).

33 As can be seen in the inset of Fig. 2(a), the $9 \mathrm{~T}$ data below $5 \mathrm{~K}$ can be well described by the Debye model $c_{p} / T=\gamma_{\mathrm{n}}$ $+A_{3} T^{2}+A_{5} T^{4}$, where $\gamma_{\mathrm{n}}$ is the normal-state electronic specific-heat coefficient and $A_{i}(i=3,5)$ denote the coefficients of the phononic contribution. The obtained parameters are $\gamma_{\mathrm{n}}=3.5 \mathrm{~mJ} / \mathrm{molK}^{2}, A_{3}=0.7 \mathrm{~mJ} / \mathrm{molK}^{4}$, and $A_{5}=0.009 \mathrm{~mJ} / \mathrm{molK}^{6}$. The $A_{3}$ value gives the Debye temperature $\Theta_{\mathrm{D}}=220 \mathrm{~K}$ via $\Theta_{\mathrm{D}}=\left(12 \pi^{4} n R / 5 A_{3}\right)^{1 / 3}$ with $n=4$ and $R=8.314 \mathrm{~J} / \mathrm{molK}$. In addition, if one assumes a single spherical Fermi surface, the effective mass $m^{*}$ can be estimated as $m^{*}=3 \hbar^{2} \gamma_{\mathrm{n}} /\left(V_{\mathrm{mol}} k_{\mathrm{B}}^{2}\left(3 \pi^{2} n_{e}\right)^{1 / 3}\right) \simeq 1.5 m_{e}$, where $V_{\mathrm{mol}}=55 \mathrm{~cm}^{3} / \mathrm{mol}$ and $m_{e}$ is the free electron mass.

${ }^{34}$ H. Padamsee, J. E. Neighbor, and C. A. Shiffman, J. Low Temp. Phys. 12, 387 (1973).

35 We fit the $B_{1}(T)$ curve with $B_{1} \propto 1 / \lambda_{\text {eff }}^{2} \propto$ $[\Delta(T) / \Delta(0)] \tanh \left(\Delta(T) / 2 k_{\mathrm{B}} T\right)$ for the local dirty limit, ${ }^{39}$ where $\lambda_{\text {eff }}$ is the effective penetration depth.

36 The positive $\chi_{0}$ is probably due to the presence of a very small amount of paramagnetic impurities.

37 N. R. Werthamer, E. Helfand, and P. C. Hohenberg, Phys. Rev. B 147, 295 (1966).

38 C.-R. Hu, Phys. Rev. B 6, 1756 (1972).

39 M. Tinkham, Introduction to superconductivity (McGraw-Hill, New York, 1975).

40 Y. Ando, G. S. Boebinger, A. Passner, L. F. Schneemeyer, T. Kimura, M. Okuya, S. Watauchi, J. Shimoyama, K. Kishio, K. Tamasaku, N. Ichikawa, and S. Uchida, Phys. Rev. B 60, 12475 (1999).

41 M. Tenhover, W. L. Johnson, and C. C. Tsuei, Solid State Commun. 38, 53 (1981).

42 P. Szabo, P. Samuely, A. G. M. Jansen, T. Klein, J. Marcus, D. Fruchart, and S. Miraglia, Physics C 369, 250 (2002).

43 P. Samuely, P. Szabo, T. Klein, A. G. M. Jansen, J. Marcus, C. Escribe-Filippini, and P. Wyder, Europhys. Lett. 41, 207 (1998).

44 L. Coffey, K. Levin, and K. A. Muttalib, Phys. Rev. B 32, 4382 (1985). 